\title{
Quaderni
}

QUADERNI Communication, technologies, pouvoir

68 | Hiver 2008-2009

Militantisme médical et fabrique des politiques de santé

\section{Médicaliser l'action en faveur de la contraception : le Planning Familial, du scandale à la stratégie de l'objectivité}

\section{Francis Sanseigne}

\section{(QpenEdition}

\section{Journals}

Édition électronique

URL : http://journals.openedition.org/quaderni/270

DOI : 10.4000/quaderni.270

ISSN : 2105-2956

Éditeur

Les éditions de la Maison des sciences de l'Homme

Édition imprimée

Date de publication : 5 janvier 2009

Pagination : 49-60

\section{Référence électronique}

Francis Sanseigne, « Médicaliser l'action en faveur de la contraception : le Planning Familial, du scandale à la stratégie de l'objectivité », Quaderni [En ligne], 68 | Hiver 2008-2009, mis en ligne le 05 janvier 2012, consulté le 30 avril 2019. URL : http://journals.openedition.org/quaderni/270 ; DOI : 10.4000/quaderni.270 


\section{$D$ ossier}

\section{médicaliser}

l'action en

faveur de la

contraception :

le Planning

Familial,

du scandale

à la stratégie

de l'objectivité

\section{Francis \\ Sanseigne}

Doctorant

GREPH-LEPS, IEP de Lyon
Un des acquis de la sociologie des problèmes publics est d'avoir montré que les situations qui paraissent «problématiques » ne le sont jamais de façon intrinsèque. Bien au contraire, elles sont perçues et qualifiées comme telles à la suite d'un travail de construction publique mobilisant des groupes, des objets, des formes rhétoriques et des chaînages argumentatifs variés au sein d'arènes multiples (Gusfield, 1981; Kitsuse et Spector, 1987). Cet article propose d'étudier certaines modalités d'établissement de la contraception en problème à résoudre et en cause à défendre en France dans les années 50 et 60 . À quoi fautil alors attribuer la modification de la loi de 1920 interdisant l'utilisation et la diffusion de tout moyen contraceptif? Nous faisons l'hypothèse que l'existence de techniques de contraception «modernes» à l'efficacité attestable (le diaphragme, puis le stérilet et la «pilule») et l'organisation illégale de leur usage, ont facilité une mobilisation en leur faveur. Ces dernières ont en effet permis au principal groupe investi dans la fabrication du "problème" (l'association «Maternité Heureuse " créée en 1956 devenue le «Planning Familial» en 1960), de produire une objectivité médicale sur les conduites contraceptives et d'édifier ainsi un argumentaire reposant sur un ensemble de motifs passés au tamis de la rationalité scientifique et de ses méthodes. La constitution de cette expertise militante, en dé-moralisant partiellement les raisons d'agir (Siméant, 2002), a favorisé l'émergence des conditions d'un langage partagé avec les formes de l'objectivité d'État. Rendre compte des effets de ce travail d'objectivation suppose de restituer à grands traits les étapes du problème et de sa carrière pour montrer les changements intervenus dans 
le registre de justification des entrepreneurs défendant la «cause contraceptive». Dans une première partie nous nous intéresserons à la façon dont cette dernière s'est instituée dans l'espace public, puis nous étudierons la façon dont elle s'y est maintenue à la faveur d'une médicalisation de l'action du « Planning Familial » qui a contribué à sa réussite.

\section{Comment naît une cause ? Ou de la nécessité du scandale}

En mars 1956, l'association « Maternité Heureuse » est créée à l'initiative de Marie-Andrée Lagroua Weill-Hallé et d'Evelyne Sullerot. Elle résulte d'une série d'actes ayant fait émerger la «cause contraceptive» au sein de l'espace public. Cette date marque l'apparition d'un groupe identifiable d'entrepreneurs en morale contraceptive (Becker, 1985, p.171-179). Elle s'inscrit dans un temps relativement bref (les quelques mois séparant le milieu de $1955 \mathrm{du}$ début de 1956) durant lequel cette question fait l'objet d'une mise en visibilité. Dans ces conditions, comment expliquer l'entrée en carrière de ce problème public qui, au milieu des années 50 , conteste la division longuement établie par la loi entre le permis et le défendu ? C'est en comparant les tentatives d'institution « ratées » à celles qui ont « réussi » que nous pourrons y répondre. Cela permettra, en outre, de comprendre la « tonalité » dominante du registre utilisé par l'association au cours de cette première période.

\section{«Un coup d'épée dans l'eau»}

C'est en ces termes que M.-A. Lagroua WeillHallé décrit ses premiers essais pour faire accéder la « cause contraceptive » à la discussion publique. Elle publie notamment le 22 mars 1953 un article dans La Semaine Médicale - journal destiné à l'ensemble de la communauté médicale - qui évoque pour la première fois le «contrôle des naissances à l'étranger et la loi française de $1920 »$. Cette interpellation reste lettre morte. L'absence de réaction publique observée chez ses pairs peut être partiellement attribuée aux faibles ressources symboliques dont la jeune gynécologue dispose. Elle conjugue en effet le discrédit lié au genre au sein d'une profession encore largement dominée par les hommes, à un capital médical propre modeste. La force persuasive d'un énoncé n'est cependant pas indépendante des formes d'énonciation choisies par le locuteur. Comme le souligne Bernard Lahire, l'effet d'un discours est imputable à des facteurs externes (contexte d'énonciation, public qui le reçoit, positions sociales respectives) et internes (formes, contenus) : par exemple, le succès d'une plaidoirie ne dépend pas uniquement de la réputation acquise par l'avocat, mais aussi de « sa forme discursive particulière (...) utilisant les techniques ou procédés des discours adéquats pour convaincre, persuader, émouvoir, ou bouleverser dans le cadre d'un tribunal» (Lahire, 2005, p.27).

L'article de 1953 se présente à la fois comme une revue documentée des expériences étrangères en matière de contraception et une histoire de la législation française sur le sujet. L'écriture est sobre : elle témoigne de la loyauté de l'auteur à l'égard de son groupe professionnel et de ses normes. Si la gynécologue mentionne «les petites brimades du personnel soignant, copiant fidèlement les médecins, brimades pouvant aller 
jusqu'au curetage sans anesthésie » (p. 152), cela ne constitue pas le cœur de son argumentation. De même, si l'introduction comporte une évocation des «drames quotidiens enregistrés (...) par les médecins accoucheurs, les chirurgiens, et les gynécologues, en ce qui concerne les conséquences désastreuses de l'avortement criminel » (p. 145), l'article ne tire aucun parti de ces situations et refuse la « ficelle» de leur dramatisation. Consciente du caractère risqué de son intervention, l'auteur se montre avant tout soucieuse de paraître en «clinicien scrupuleux vis-à-vis de la loi » (p. 150). C'est la raison pour laquelle elle cherche à offrir toutes les garanties rhétoriques de sérieux et endosse le rôle d'une collègue s'adressant à ses semblables sur une question concernant leurs pratiques professionnelles communes. Cette recherche de crédit a cependant un effet paradoxal : la dénonciation contenue implicitement dans l'article n'est pas en mesure d'ouvrir une succession d'échanges publics sur le sujet. Dans ce premier moment, tout se passe comme si la crainte de voir se transformer en un stigmate durable l'illégitimité et l'illégalité potentielle du texte et de son contenu, interdisait à la jeune gynécologue de recourir à des formes capables d'attirer l'attention de ses pairs par des techniques de visibilisation efficaces, c'est-à-dire destinées à « faire apparaître certains faits, ou certaines relations entre des faits, qui n'était pas à proprement parler cachés mais seulement indistincts et indisponibles comme tels » (Lemieux, 2008, p.137). En outre, l'arène choisie pour aborder la question limite ses chances d'institution. Le public y est en effet restreint, majoritairement conservateur, et ne permet guère d'espérer un recrutement d'alliés suffisants pour modifier une législation concernant différents collectifs.

\section{Produire un scandale}

Un scandale apparaît lorsqu'une contradiction entre des situations vécues et des valeurs d'un groupe fait l'objet d'un travail de désignation publique (De Blic et Lemieux, 2005). Après l'échec décrit précédemment, cette stratégie de l'épreuve sera principalement employée par les pionniers de la «cause contraceptive » entre 1955 et 1960 . Afin de produire un scandale capable d'instituer cette dernière, la scène retenue ne se limite plus au monde médical. Ce sont les journaux généralistes et les livres qui permettent tout à la fois de constituer et d'accéder à un public élargi en mesure d'être enrôlé. Les techniques de mobilisation reposent sur l'exploitation de «cas frappants » (Lahire, 2005, p. 144) : en proposant au lecteur une identification à distance, elles cherchent à s'appuyer sur des émotions partageables pour s'attacher le plus grand nombre. De ce point de vue, il n'est pas étonnant que la carrière du problème " prenne » réellement à la faveur d'un procès (celui, en 1955, des époux Bac), fournissant une occasion d'illustrer les conséquences « dramatiques » de la législation. En outre, le partage du sensible est facilité par le sujet lui-même, qui offre la possibilité de mettre publiquement en scène des corps meurtris et de produire une forme d' " intolérable » (Bourdelais et Fassin, 2005). Enfin, le registre du scandale utilisé à cette période comporte cette fois une structure dénonciatrice explicite empruntant des formes qui la manifestent (Boltanski, Darré et Schlitz, 1984). On y retrouve l'exigence de désingularisation des « victimes » (les « femmes », le « couple », la « famille ») et des « coupables » (la « loi », l' « hypocrisie » collective), condition nécessaire pour être reçu dans l'espace public. 
Ainsi, par la mise en circulation d'émotions au moyen de l'exposition de cas, le but est de s'adresser au plus grand nombre, de « frapper les esprits » en renvoyant chacun à son expérience personnelle. Il s'agit en même temps de référer à des valeurs instituées (la justice, l'humanité) présentées comme bafouées. Ce sont ces diverses propriétés que possèdent les articles du journaliste Jacques Derogy parus à l'automne 1955 et l'ouvrage La Grand Peur d'Aimer de M.-A. Lagroua Weill-Hallé qui, lors de leur parution respective, rencontrent un vif retentissement.

Les huit articles de J. Derogy sont publiés dans le quotidien communisant Libération sous le titre "Les femmes sont-elles coupables? ». C'est à la suite de sa rencontre avec M.-A. Lagroua Weill-Hallé que le journaliste entreprend de les rédiger. L'auteur y dénonce le « véritable fléau » de l'avortement qui plonge des femmes dans une clandestinité périlleuse. Les trois premiers articles sont ainsi consacrés au spectacle des souffrances résultant des grossesses que l'on interrompt et des « drames » domestiques noués autour d'enfants non voulus. Le premier chapeau constate : «Seules, ou entre des mains hésitantes, des malheureuses, affreusement torturées risquent la mort sans le savoir » (17 octobre); les sous-titres révèlent un « drame innombrable » (17 octobre), une «réalité bouleversante» (18 octobre), «deux cadavres par semaine » (19 octobre). Au lecteur rien n'est épargné : le journal le conduit volontiers dans les salles d'hôpitaux («derrière la façade », 17 octobre) où des femmes mutilées sont accueillies après qu'elles ont avorté ; au fil des colonnes, les portraits de « victime» de la législation se multiplient. Après une présentation de la situation étrangère, le jour- naliste désigne publiquement les coupables sous le titre : « responsable du fléau des avortements : la Loi ! » (22 et 23 octobre). À côté de la figure $\mathrm{du}$ " législateur », une autre entité collective fait l'objet d'une imputation en responsabilité : «la rigueur intransigeante de la doctrine catholique» (25 octobre). Par sa rhétorique de la dramatisation, par les coups de projecteur qu'il fait sur des situations officieuses, J. Derogy contribue fortement à la scandalisation de la question. En outre, ces articles énoncent avec clarté ce qui formera l'un des principaux credos de la période : la contraception « moderne », bien qu'interdite, constitue le « remède » au « fléau » de l'avortement. Sa dénonciation publique frappe, qui est à l'origine de vives discussions dans la presse les mois suivants. Les articles seront repris sous la forme d'un livre intitulé Des enfants malgré nous, qui paraîtra en 1956 aux Éditions de Minuit avec une préface de M-A Lagroua Weill-Hallé.

En 1960, cette dernière publie à son tour un livre. Son nom n'est pas inconnu du public, notamment en raison de la création de « Maternité Heureuse ». Cependant, l'écho que l'ouvrage rencontre, le rôle qu'il joue dans l'institution de la « cause contraceptive », doivent être attribués au registre mobilisé. Le texte se présente en effet comme le «journal d'une femme médecin». Il comporte ainsi un pacte de lecture de nature autobiographique (Lejeune, 1996): le contrat passé avec les attentes du lecteur suppose la référentialité (ou plutôt l'entretien de son illusion). Ce qui est écrit doit alors correspondre à la réalité, à des choses «vues et entendues » par celui ou celle qui les rapporte. Cette présomption de réel est également renforcée par une injonction à l'authenticité et à la 
sincérité que le genre requiert. Elle contribue au sentiment du lecteur d'avoir accès à la « vérité » et au «vécu » d'une personne parlant en son nom propre. Avec La Grand'Peur d'Aimer, le contrat est respecté, qui met en œuvre un véritable dispositif d'exposition de «tranches de vie ». Après avoir fait le récit exemplaire de sa conversion à la cause contraceptive (on y retrouve notamment la narration détaillée des peines endurées par une femme ayant avorté et qui, une fois le curetage à vif subi, est laissée à l'abandon dans une arrière-salle d'hôpital), l'auteur ouvre les portes de son cabinet. Elle procède alors à une accumulation sensible de cas plutôt qu'à une étude, objectivant de façon systématique les difficultés rencontrées par sa clientèle. Des vignettes de longueur variable se succèdent selon une même organisation: une fois brossé le portrait de la personne venue consulter, le lecteur assiste aux échanges verbaux avec la gynécologue à propos de la maternité et de ses difficultés. Ces interactions et les attestations de vérité qui les accompagnent (les sanglots, les cris, la description de corps fatigués ou meurtris sont récurrents) sont parfois associées à un titre qui suggère l'extrême variété des situations ("Quand un rationaliste perd les pédales», "Quand les femmes catholiques se font avorter», "Il y aussi celles qui ont une carrière », «On n'en parle jamais voilà», etc.). Ces mises en récit de la douleur physique et morale se concluent généralement par un commentaire où la diariste communique sa stupéfaction, son impuissance («Je me suis sentie d'une inefficacité déplorable», p.36) ou son indignation ( Quatre enfants qui accaparent tout de ce corps et de cette âme, quatre enfants et un père nourricier! Quelle situation mal équilibrée ! Et tout cela au nom d'une morale! C'est donc ça le devoir ?», p.99). Ce dispositif de mobilisation et d'enrôlement à distance repose ainsi sur l'impression et l'émotion, dans le but d'établir les conditions d'un scandale («Leur cri [des femmes] de détresse ne peut plus rester étouffé par une certaine forme de Morale », p.28). De plus, à côté de sa fonction de recommandation, la préface de Simone de Beauvoir accentue volontairement le caractère dénonciateur de l'ouvrage. En conséquence, par ces différents aspects, celui-ci est loin de la bienséance professionnelle qui marquait l'article de 1953. S'il forme une contribution essentielle à la transformation de la contraception en problème public, c'est parce qu'il parvient à instituer un groupe élargi de « victimes » dont la parole peut susciter la colère et/ou une identification, faire scandale, et finalement établir la possibilité d'un changement.

\section{Faire tenir et se maintenir: la mise en objectivité de la « cause contraceptive »}

À la façon du charisme weberien, une cause qui paraît dans l'espace public rencontre le problème de son entretien. La transformation (partielle) du répertoire d'action de «Maternité Heureuse » peut être attribuée à deux facteurs. Se maintenir dans le seul registre de l'indignation suppose de produire des « coups » (éditoriaux ou autres) à répétition qui, à force de s'appuyer sur le régime des émotions, s'exposent au risque de rendements décroissants ou perçus comme tels ; ce qui ne signifie pas que, à partir de 1960, les interventions d'éclat cessent, mais qu'elles sont d'une autre nature. Cette modification doit être également mise en rapport avec l'importance que 
prend le groupe des médecins dans l'association et sa direction. Elle correspond ainsi à la valorisation d'un avantage comparatif (Contamin, 2005) qui les conduit à produire des savoirs et des pratiques réglées sur les canons de l'objectivation scientifique, conformément à leur ethos professionnel. La médicalisation de la question de la contraception (contre l'avis de l'Ordre des médecins) leur permet alors de former un monopole de connaissances constitué par l'enquête qui, tout à la fois, peut concurrencer et consoner avec l'objectivité d'État.

\section{Une objectivité à soi}

Dans la préface à Des enfants malgré nous, M.-A. Lagroua Weill-Hallé déplorait que le «drame » de l'avortement fût à cette date mieux connu des romans et des mises en forme sensible qu'ils fabriquent, que du monde médical. Une décennie plus tard, dans l'introduction d'un ouvrage destiné à ses pairs, la fondatrice du « Planning Familial » résume son action en ces termes : «le monde médical était si pusillanime en 1955 à propos de la contraception, qu'il eût été illusoire de ne s'adresser qu'à lui pour modifier son attitude au regard de ce problème. Moi-même j'ai été mêlée (...) à ce problème d'opinion, mais très vite j'ai compris que seule, l'observation médicale poursuivie scientifiquement et d'une manière prolongée parviendrait à lever les interdits » (Lagroua Weill-Hallé, 1968, p. 12). Cette croyance dans les effets de l'argument scientifique est collectivement partagée par les entrepreneurs de la période, au-delà des trajectoires (tous ne sont pas médecins) et des raisons variées de s'investir. Le credo constamment répété selon lequel la contraception serait le « remède » à l'avortement ne peut se durcir que si l'efficacité des techniques contraceptives est certifiée sous le rapport des bénéfices physiques et moraux. C'est dans ce but que l'enquête empirique comme style de raisonnement et de pratiques est mobilisée au cours des années 60 : il s'agit autant de stabiliser des savoir-faire médicaux que d'objectiver les personnes ayant eu recours à la contraception pour en connaître directement les effets.

De ce point de vue, le colloque organisé à Royaumont en 1963 est une manifestation publique des premiers résultats accumulés par le «Planning Familial». Les interventions qui s'y succèdent sont les indices de la façon dont le problème a cristallisé au sein de l'association. Ainsi la deuxième journée est-elle consacrée à son «aspect médical et [aux] réalisations pratiques $» . \mathrm{La}$ présentation d'informations « objectives » sur les moyens contraceptifs s'inscrit dans le cadre de la mutualisation d'expériences de plusieurs médecins. Le contraste avec les années précédentes provient du fait que les données présentées sont pour une bonne part originales. $\mathrm{Si}$, dans la période antérieure, M.-A. Lagroua Weill-Hallé avait bel et bien publié deux ouvrages à l'audience restée modeste sur des aspects techniques du contrôle des naissances, leur contenu résultait d'une circulation de savoirs élaborés essentiellement dans le monde anglo-saxon ${ }^{1}$. L'édification de connaissances indigènes indexées sur le «terrain » français est la conséquence d'un changement important dans l'action du « Planning Familial ». En ouvrant en 1961 une permanence où des contraceptifs « modernes » sont illégalement diffusés, le groupe de Grenoble réalise un « coup » tactique qui aura trois effets directs. D'une part, devant le succès de l'initiative, la direction 
encourage sa multiplication. D'autre part, il entraîne un accroissement important des effectifs : l'obtention de moyens contraceptifs est en effet conditionnée par l'adhésion ; cela permet ainsi de se garantir contre l'accusation de «propagande anticonceptionnelle », tout en renforçant le poids numérique de l'association et ses ressources. Enfin, cet afflux soudain a établi les conditions d'une collecte rigoureuse d'observations sur une population élargie. Aussi, cette objectivité à soi qui expérimente des connaissances « étrangères » et fabrique des savoirs et des savoir-faire locaux, contribue à une forme de «nationalisation » du répertoire des preuves utilisé. Dans la rhétorique publique déployée, la démonstration de l'intérêt de la contraception peut ainsi se soutenir d'une compétence technique acquise et transmise au contact de la réalité française.

Dans ses mémoires, Pierre Simon, un des premiers médecins-hommes à avoir rejoint l'association, souligne ainsi : «l'enseignement est indispensable pour éduquer d'autres médecins à cette pratique de façon à inscrire dans le champ du réel ce que rejette la loi »(Simon, 1979). En 1962, la création du Collège des Médecins (Garcia, 2006) qu'il préside, illustre également ce processus : face à la nécessité de structurer le mouvement sous le rapport de la prescription et de l'utilisation de contraceptifs, une formation est mise en place qui contribue à standardiser les pratiques de médecins (mais aussi celles des « hôtesses d'accueil »). À l'apprentissage sur le « tas » revêtu des charmes de la parole et du geste délivrés entre collègues au cours d'interactions personnelles, succède une transmission élargie nécessitant un travail supplémentaire d'objectivation, en particulier par la constitution de documents écrits.
Comment l'objet « contraception » est-il construit dans les enquêtes favorisées par cette nouvelle configuration ? Le point commun entre les observations de M.-A Lagroua Weill-Hallé, celles effectuées au sein d'un important centre d' « orthogénie » ouvert par la MGEN² en 1962, ou encore celles issues du travail d'Anne-Marie Dourlen-Rollier sur les avortements clandestins, réside dans l'usage systématique de la mise en chiffre, le recours au langage tabulaire, c'est-àdire aux différentes manifestations de la « raison graphique » (Goody, 1979). L'enquête ne consiste plus en un recueil impressionniste de cas ; au contraire, elle s'emploie à produire une mesure rigoureuse de la question contraceptive par le codage systématique de situations rencontrées dans l'univers médical ou ailleurs ${ }^{3}$. Dans les deux premiers exemples, la pratique de la « fiche d'observation » conjuguée à celle du questionnaire permet d'abstraire les personnes de la richesse inépuisable des contextes dans lesquelles elles sont encastrées. Ces instruments de rupture avec l'expérience singulière saisissent les conduites contraceptives d'une façon double : d'une part, en caractérisant la population étudiée de façon stylisée sous le rapport de l'âge, de la profession, de son « niveau culturel », de sa religion, de son état matrimonial ou de son «désir d'enfant»; d'autre part, en mesurant les avantages des nouvelles méthodes proposées tant au niveau de leur commodité que des effets sur le « couple» et sa « vie sexuelle», que l'on peut croiser avec les premières variables. Cet investissement de forme (Thévenot, 1985) va être sophistiqué au fil des années ${ }^{4}$; cela autorisera le « Planning Familial » à revendiquer la propriété de connaissances qui «tiennent» parce que celles-ci comportent une présomption d'objectivité imputable aux 
procédures de quantification, délestant en partie la cause du poids de la dénonciation ou de la politisation. Ainsi les entrepreneurs vont-ils être en mesure de constituer une sorte d'évidence partageable avec certains modes étatiques de raisonnement.

\section{Concurrence et Résonance}

Comme l'ont montré de nombreux travaux ${ }^{5}$, la construction de l'État est fortement liée au développement d'une instrumentation statistique. Celle-ci met en œuvre une description quantifiée de phénomènes rendus commensurables pour décider et administrer. Bien souvent, défendre une cause suppose de contester des routines cognitives et des pratiques solidifiées au sein d'une configuration donnée à la suite de transactions nombreuses entre groupes divers. Dans notre étude, plaider en faveur d'une législation nouvelle suppose la mise en question du point de vue de l'État, lequel est en grande partie informé par l'entendement démographique. Toutefois, la stratégie retenue ici n'est pas celle d'une subversion intégrale ou revendiquée comme telle. Alors que l'on constate une concurrence entre formes d'objectivité, celles-ci ont en commun un travail d'objectivation qui rend possible, sinon un accord, au moins une "résonance de cadres" (Benford et Snow, 1986).

Si la notion de « population » apparaît au XVII ${ }^{\mathrm{e}}$ siècle, sa signification n'est pas univoque ; ce terme fait l'objet d'appropriations par des groupes et des savoirs variés. Dans le cas français, la formation d'un véritable « regard » démographique date du milieu du XIX ${ }^{\mathrm{e}}$ siècle (Schweber, 2006). Toutefois, son institutionnalisation au sein du monde politico-administratif ne se produit qu'à la fin des années 40 (Rosental, 2003). L'Institut National d'Études Démographiques (INED) est créé en 1945 : placé sous la direction d'Alfred Sauvy, il atteste l'existence d'un réseau de personnes et d'institutions stabilisé à la Libération mais initié dans les années 30. Sa vocation est alors d'offrir une « intelligence démographique », c'est-à-dire une « construction simultanée qui unit théories et politiques démographiques » (Rosental, 2003, p. 140). Rien d'étonnant à voir l'INED intervenir armé de ses propres travaux au sujet de la « régulation des naissances », ou encore de le voir sollicité par le gouvernement sur cette question au côté du Haut comité consultatif pour la population et la famille. Comme les articles publiés dans la revue Population par ses membres en témoignent, ce « terrain » y est tôt investi. Cependant, il repose sur les principes d'une démographie pure qui entend constituer un point de vue sui generis sur la «population». Celui-ci cherche alors à définir les lois de son évolution à partir de facteurs présumés internes comme la « fécondité », la « mortalité », ou encore la «nuptialité ». Par conséquent, ce style de raisonnement introduit une entité nouvelle descriptible sous le rapport des seules variations quantitatives. En raison de la position occupée par la démographie comme savoir d'État et les techniques d'objectivation qu'elle emploie, il n'est guère surprenant de voir ses représentants défendre publiquement une forme de natalisme exclusivement soucieux de l'intérêt de la « population » alors conçue comme une réalité réifiée. De ce point de vue, l'article paru en 1956 dans Population au moment de l'institution publique de la cause contraceptive est exemplaire par les sérieuses réserves qu'il émet à l'égard de tout changement législatif au 
nom du « seul élément mobile et objectif, (...) les données démographiques» (p. 213).

L'objectivité revendiquée par les entrepreneurs $\mathrm{du}$ «Planning Familial» emprunte une échelle d'observation différente. L'argument démographique y est certes présent, qui se trouve au principe d'une querelle en nomination concernant la réalité recouverte par les moyens contraceptifs : s'agit-il en effet d'une «limitation» des naissances aux échos malthusiens ? ou, alors, d'une "planification », d'un « contrôle », d'un « espacement » de cellesci, davantage compatible avec le «bon sens » démographique? Néanmoins, cette question n'occupe pas la première place dans la fabrique d'un répertoire de preuves. La réponse apportée à la «menace» du déclin démographique ne l'est pas en fonction de la grammaire nombre. Comme nous l'avons montré, la contraception est saisie du point de vue des individus ou du couple, de leurs comportements, des situations dans lesquelles ils se retrouvent, de son efficacité, et des pratiques médicales qu'elle occasionne. Habitués par leur formation au colloque singulier, les médecins du «Planning Familial » sont disposés à voir le problème à hauteur de femmes ou d'hommes, plutôt que sous le rapport de la «population». De plus, la médicalisation de la question est justifiée par la notion de "santé » élaborée par l'OMS en 1947 : celle-ci ne désigne pas une absence de maladie, mais un état de «bien-être physique, mental, et social» auquel chacun à droit, ce qui n'incite pas à valoriser le point de vue du collectif par rapport à celui de l'individu. Le travail d'objectivation entrepris par l'association est cependant original : il repose sur la mise au point d'une forme de médecine proche des raisonnements de santé publique s'appuyant sur une description statistique élémentaire des personnes, de leurs motivations, et des contextes dans lesquelles elles sont inscrites, à une époque où ce type d'approche n'a pas acquis ses lettres de noblesse dans le monde médical français. C'est sur ce point que les formes d'objectivité militante et d'État peuvent résonner. La recherche de robustesse et de crédit de la première, rencontre certains aspects des techniques cognitives de l'autre. Un indice pourrait en être trouvé dans les formes de concurrence à distance, voire des tentatives de collaboration, existant entre les recherches du Planning Familial et celles de l'INED. Pour ces dernières, le recours à l'instrument « sondage » trouve un de ses premiers terrains d'application sur les questions liées à la «natalité » ou la « maternité » (Blondiaux, 1998, p. 444-446). Il s'agit d'introduire la prise en compte des « opinions individuelles» au cœur de la démographie. Les enquêtes menées à Paris, Lyon, Grenoble ou auprès d'un «échantillon de la population française », sont des réponses explicites à celles conduites par le «Planning Familial ». Si elles prétendent les corriger, les confirmer ou les infirmer sur certains points (et réciproquement), elles partagent des cadres communs. La cause défendue par les entrepreneurs de l'association voit ainsi ses chances de succès s'accroître en même temps qu'elle prend des formes énonçables suivant un « air de famille» (Wittgenstein, 1961, § 67) partagé avec l'objectivité d'État, sans toutefois s'y confondre. En effet, si les enquêtes menées par des membres de l'association se démarquent du régime de l'accumulation sensible en 
recourant à la quantification, elles ne se placent pourtant pas au niveau de la «population» telle que l'entend ordinairement « l'intelligence démographique $»$.

\section{Conclusion}

Comment estimer les bénéfices apportés par la stratégie de l'objectivité à la défense de la « cause contraceptive » et à la résolution des « problèmes » qu'elle désigne publiquement? Sans postuler l'existence d'un facteur explicatif « en dernière instance », il est possible d'attribuer à celle-ci un rôle essentiel. Une fois la cause instituée au moyen du scandale, le processus de sa médicalisation a conduit le « Planning Familial » à disposer d'un double monopole de connaissances issu de son travail d'enquête : à propos de la contraception elle-même sous ses aspects techniques et pratiques, d'abord ; sur les personnes et concernant les contextes dans lesquels la demande contraceptive était formulée, ensuite. Parce qu'ils possédaient une affinité de forme avec l'objectivité d'État, ces savoirs et savoirfaire ont contribué à rendre possible l'acceptation de la « cause contraceptive » en accroissant son crédit et en durcissant son argumentaire face aux catégories démographiques de la pensée d'État. Dès lors, munis des ces ressources, il n'est pas étonnant que certains membres de la première génération du «Planning ${ }^{6}$ se soient retrouvés au cours des années 70 dans le monde des cabinets ministériels ou dans différents organismes aux fortunes diverses en rapport avec la contraception, l'avortement, l'éducation sexuelle, afin d'y faire valoir une expertise d'origine militante compatible avec un souci de santé publique.

\section{$R \cdot E \cdot F \cdot E \cdot R \cdot E \cdot N \cdot C \cdot E \cdot S$}

BECKER Howard, (1985), Outsiders, Paris, Métailié.

BENFORD Robert et SNOW David, (1988), « Ideology, frame resonance and participant mobilization » in International Social Movement research, ${ }^{\circ} 1$, pp. 197-217.

BLONDIAUX Loïc, (1998), La fabrique de l'opinion, Paris, Le Seuil.

BOLTANSKI Luc, DARRÉ Yann et SCHILTZ Marie-Ange, (1984), «La dénonciation » in Actes de la recherche en sciences sociales, $\mathrm{n}^{\circ} 51$, pp. 3-40.

BOURDELAIS Patrice et FASSIN Didier (dir.), (2005), Les constructions de l'intolérable, Paris, La Découverte.

CONTAMIN Jean-Gabriel, (2005), «Le choix des armes : les dilemmes pratiques d'un mouvement de doctorants et le modèle des avantages comparatifs » in Genèses, $\mathrm{n}^{\circ} 59$, pp. 4-24.

DESROSIÈRES Alain, (1993), La politique des grands nombres, Paris, La Découverte.

De BLIC Damien et Cyril LEMIEUX Cyril, (2005), «Le scandale comme épreuve» in Politix, n71, 9-39.

DOURLEN-ROLLIER Anne-Marie, (1963), La vérité sur l'avortement, deux enquêtes inédites, Paris, Maloine.

GARCIA Sandrine, (2006), «La création du collège des médecins : un coupe de force symbolique » in Christine Bard et Janine Mossuz-Lavau (dir.), Le Planning Familial, histoire et mémoire, Rennes, PUR, pp. 109-117. GOODY Jack, (1979), La raison graphique, Paris, Éditions de Minuit. 
GUSFIELD Joseph, (1981), The culture of public problems : drinking-driving and the symbolic order, Chicago, The University of Chicago Press. KITSUSE John et SPECTOR Malcolm, (1987), Constructing social problems, New York, Aldine de Gruyter.

LAGROUA Weill et HALLÉ Marie-Andrée, (1968), La prescription contraceptive, Paris, Maloine.

LAHIRE Bernard, (2005), L'invention de " l'illettrisme », Paris, La découverte.

LEMIEUX Cyril, (2008), « Rendre visibles les dangers du nucléaire. Une contribution à la sociologie de la mobilisation » in Bernard Lahire et Claude Rosental (dir.), La cognition au prisme des sciences sociales, Paris, Éditions des Archives Contemporaines.

LEJEUNE Philippe, (1996), Le pacte autobiographique, Paris, Points Seuil.

ROSENTAL Paul-André, (2003), L'intelligence démographique, Paris, Fayard.

SCHWEBER Libby, (2006), Disciplining statistics, Durham, Duke University Press.

SIMÉANT Johanna, (2002), « Friches, hybrides, et contrebandes : sur la circulation et la puissance militantes des discours savants » in Hamman Philippe, Jean-Mathieu Méon et Benoît Verrier (dir.), Discours savants, discours militants Paris, L'Harmattan, pp. 17-53.

SIMON Pierre, (1979), De la vie avant toute chose, Paris, Mazarine.

THÉVENOT Laurent, (1985), «Les investissements de formes » in Cahiers du CEE, n²9, pp. 21-71.

WITTGENSTEIN Ludwig, (1961), Investigations philosophiques, Paris, Gallimard.
$\mathrm{N} \cdot \mathrm{O} \cdot \mathrm{T} \cdot \mathrm{E} \cdot \mathrm{S}$

1. La fondatrice du « Planning Familial » a découvert le «birth control» lors d'un voyage aux ÉtatsUnis. Par la suite, pour les premiers médecins de l'association, le pèlerinage en terre britannique devient un passage obligatoire.

2. Celui-ci a des liens étroits avec le «Planning Familial » puisque deux des médecins qui y officient, Christiane Verdoux et Jacqueline Kahn-Nathan, font également partie du groupe dirigeant de l'association pendant les années 60 .

3. Anne-Marie Dourlen-Rollier enquête sur les avortements clandestins sur la base de dossiers judiciaires et médicaux. Voir: (Dourlen-Rollier, 1963).

4. Pour s'en convaincre, on pourrait comparer l'enquête de M.-A. Lagroua Weill-Hallé parue dans Gynécologie Pratique en 1960 à celle, en forme de bilan, publiée dans l'ouvrage La contraception et les Français, enquête sur 7600 couples.

5. Pour s'en tenir à la littérature française, voir l'ouvrage classique d'Alain Desrosières, La politique des grands nombres, Paris, La Découverte, 1993.

6. Celle entrée à la fin des années 50 et qui quitte l'organisation au début des années 70 à la faveur de sa radicalisation politique. 
$R \cdot E ́ \cdot S \cdot U \cdot M \cdot E ́$

Cet article propose d'étudier certaines modalités d'établissement de la contraception en problème à résoudre et en cause à défendre en France dans les années 50 et 60 . Il fait l'hypothèse que l'existence de techniques de contraception « modernes » à l'efficacité attestable et l'organisation illégale de leur usage, ont contribué à la réussite de la mobilisation en leur faveur que le changement de la législation traduit. Ces deux facteurs ont en effet permis au « Planning Familial » de produire une objectivité médicale sur les conduites contraceptives et d'édifier un argumentaire reposant sur des savoirs fabriqués par la pratique de l'enquête et le déploiement de la rationalité scientifique. Parce qu'ils possèdent une affinité de forme avec l'objectivité d'État qui, en la matière, s'est construite autour du « regard» démographique, ces savoirs et savoir-faire ont contribué à rendre possible l'acceptation de la « cause contraceptive » en accroissant son crédit et en instituant les conditions d'une évidence partagée.

This paper details some ways by which birth control became a problem to solve and a cause to support in France during the 50's and the 60's. Our hypothesis is that the effectiveness of new contraceptive devices as well as their planned illegal use, have made possible a successful mobilization towards a change of law. These two factors enabled the French Family Planning Association to produce a medical objectivity concerning contraception practices and to build strong arguments relying on the use of knowledge provided by inquiry and scientific rationality. Because of its formal affinity to the State objectivity which frames the issue with a demographical eye, this knowledge made the recognition of the contraception claim possible by a raise of its credit and by setting the conditions of a shared evidence. 